Ann. Biol. anim. Bioch. Biophys., I970, 10 (I), I5I-I57.

\title{
MÉTHODE DE DOSAGE DES ACIDES GRAS VOLATILS DANS LE LIQUIDE DU RUMEN
}

\author{
J. RIGAUD et M. JOURNET \\ avec la collaboration technique de Jeanne FLEcheT \\ Station de Recherches sur l'Élevage des Ruminants, \\ Centre de Recherches de Clermont-Ferrand, 63-Saint-Genès-Champanelle \\ Institut national de la Recherche agronomique
}

RÉSUMÉ

Nous proposons une méthode rapide de dosage des acides gras volatils, dans le liquide du rumen, par chromatographie en phase gazeuse. A l'échantillon filtré grossièrement, on ajoute de l'acide formique et du butanol comme étalon interne. L'analyse est effectuée en I 5 minutes sur deux colonnes mises bout à bout et composées, l'une de Carbowax $20 \mathrm{M}$ sur Chromosorb, l'autre de Porapak T. L'addition d'acide formique au gaz vecteur en même temps qu'à l'échantillon supprime pratiquement l'adsorption. La méthode permet de déterminer avec précision les proportions des différents acides et d'évaluer leur concentration grâce au butanol. Elle sépare très bien l'acide isobutyrique. Elle exige certaines précautions qui sont détaillées à la fin du texte.

\section{INTRODUCTION}

L'étude de l'orientation des fermentations dans le rumen nécessite de connaitre la concentration en acides gras volatils (A.G.V.) totaux et les proportions de ces différents acides: acétique, propionique, isobutyrique, butyrique, isovalérianique et valérianique. Ceci donne généralement lieu à deux opérations distinctes, respectivement une distillation et un fractionnement par chromatographie en phase gazeuse.

I 1 es méthodes classiques de distillation, dérivées de celle de FRIEDMANN (I938), sont trop longues pour être adoptées dans les dosages en grande série. VANDENHEUVEI. (I964) a proposé une méthode rapide et précise de microdistillation que nous avons utilisée jusqu'ici après l'avoir modifiée. Elle ne dose pas spécifiquement les acides gras volatils, mais tous les acides volatils y compris l'acide formique en presque totalité (90 p. IOO) et une petite fraction de l'acide lactique. Ces acides représentent de o à 8 p. Ioo des acides totaux selon les échantillons de jus de rumen. Dans l'étude qui 
suit nous avons cherché, à la suite de nombreux auteurs, à supprimer la distillation et à ramener les deux opérations du dosage à une simple chromatographie en phase gazeuse.

La première méthode de déternination de la composition d'un mélange d'acides gras volatils par chromatographie en phase gazeuse mise au point par JAMEs et MARTIN (I952), a été utilisée pendant plusieurs années dans notre laboratoire. Mais cette méthode était longue puisque le fractionnement proprement dit durait 50 minutes et qu'il ne pouvait s'effectuer à partir d'une solution aqueuse. Pour mettre les acides gras volatils en solution organique, nous avions recours à la technique d'ELSDEN (I946). Diverses autres techniques, visant aussi à éviter l'introduction d'eau sur la colonne, furent proposées (Grhrke et Lamkin, ig6r; 'Tiliey, Canaway et Terrry, I964; StORRY et MiliaRd, ig65). Bien que plus rapides que celle d'ElsDen, elles contribuent à allonger nettement la durée des opérations. Monk et ForREST (1967) injectent le liquide du rumen centrifugé sur une " précolonne " remplie d'un mélange absorbant l'eau, mais nous avons pu vérifier que ce procédé avait pour conséquence l'obtention de pics plus larges et asymétriques. Les méthodes basées sur l'estérification des acides (FABRY, I967, cite 33 de ces méthodes) permettent d'éliminer l'eau et de supprimer la traînée des pics; mais elles introduisent aussi une manipulation supplémentaire et peuvent conduire à des pertes d'acides gras volatils, même si le rendement de la réaction est bon, à cause de la volatilité des esters. Nous nous sommes donc orientés vers les méthodes permettant l'injection du liquide du rumen sur la colonne, seules compatibles avec les impératifs de rapidité et de simplicité que nous nous étions fixés.

Un des premiers auteurs qui ait fractionné, par chromatographie en phase gazeuse, un mélange d'acides gras volatils en solution aqueuse est HunTeR (I960) ; mais la quantité d'eau admise ne pouvait excéder 50 p. Ioo de l'échantillon et les pics, que lui donnaient ces colonnes à base de silicones, présentaient des traînées telles que leur surface n'était pas mesurable. L'utilisation d'une phase stationnaire, le Tween 80, a permis à EMrRY et KOERNER (Ig6I) d'analyser des solutions aqueuses diluées d'acides gras volatils, puis à FRWIN, MARCo et EMERY (I96I) de travailler à partir du liquide de rumen lui-même. Depuis lors, de nombreuses autres méthodes voisines ont été proposées, mais à notre connaissance, deux problèmes importants n'avaient pas trouvé de réponse satisfaisante :

- les colonnes suffisamment stables en présence d'eau ne permettent pas une séparation rapide et correcte de tous les acides gras volatils qui nous intéressent;

- l'adsorption des acides gras volatils fausse l'estimation de la composition du mélange puisque l'élution n'est pas quantitative. Si l'on veut obtenir des résultats valables, il faut faire des injections répétées du même échantillon (VAN EENAEME, BIENFAit, I IAMBOT, I965), ou des injections d'eau entre les analyses (MAHADEVAN et STENROOS 1967), ce qui diminue d'autant les possibilités de travail.

Nous pensons avoir surmonté ces difficultés et nous proposons une méthode qui permet d'évaluer en même temps le pourcentage des différents acides gras volatils et leur concentration dans le liquide du rumen. 


\section{CHOIX D'UNE MÉTHODE}

En ce qui concerne les colonnes nous avons essayé, sans qu'aucune ne donne satisfaction, la plupart des phases stationnaires utilisées par les autres auteurs. Nous nous sommes attachés à obtenir une bonne séparation de l'acide isobutyrique qui se trouve en quantité non négligeable dans la plupart des contenus du rumen. Nous avions remarqué que sur une colonne de Carbowax $20 \mathrm{M}$, son pic chevauchait en partie celui de l'acide propionique, alors que sur une colonne de Porapak $T$, il était presque confondu avec celui de l'acide butyrique. Nous avons alors pensé qu'il serait intéressant de mettre bout à bout ces deux types de colonnes et après avoir essayé différentes longueurs, nous sommes arrivés au résultat illustré par la figure I.

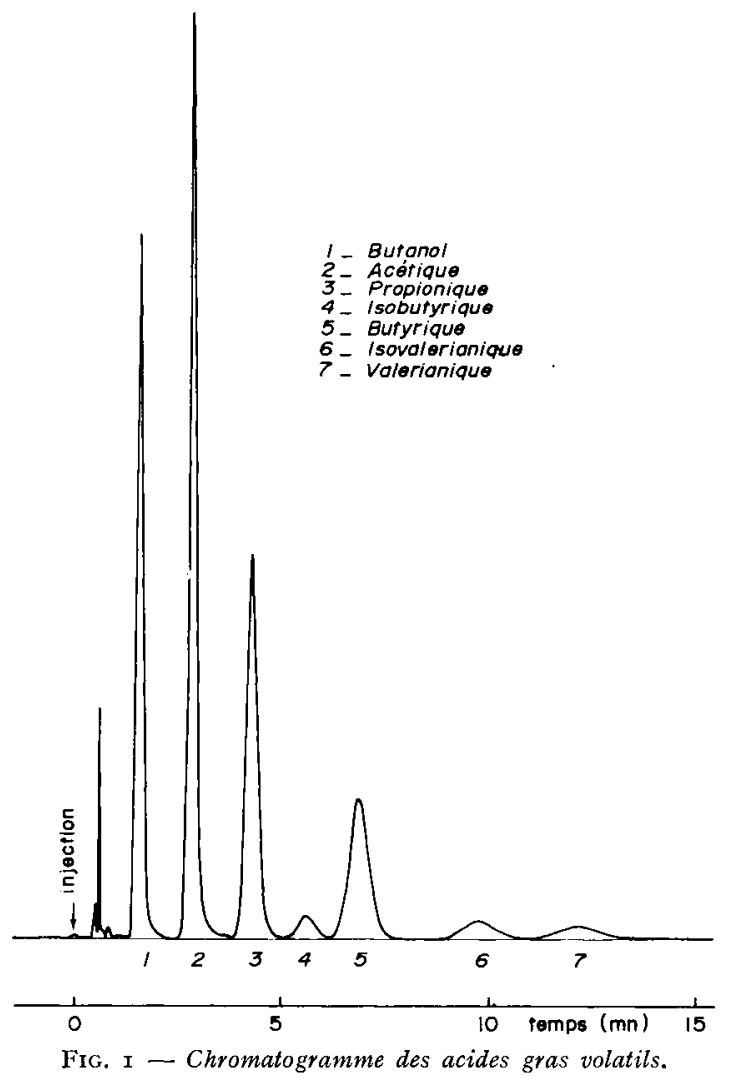

Le bon fractionnement obtenu permet d'effectuer l'analyse en moins de I5 minutes et les résultats sont reproductibles, comme nous avons pu le vérifier en analysant ro fois le même échantillon d'un liquide du rumen sur les 2 colonnes A et B de l'appa- 
reil constituées chacune d'une colonne de Porapak $\mathrm{T}$ et d'une colonne de Carbowax $20 \mathrm{M}$.

\section{Colonne A Colonne B}

\begin{tabular}{|c|c|c|}
\hline & - & - \\
\hline Acétique & $63,8 \pm 0,4$ & $63,6 \pm 0,4$ \\
\hline Propionique... & $23,6 \pm 0,3$ & $23,7 \pm 0,3$ \\
\hline Isobutyrique & $0,8 \pm 0,2$ & $0,9 \pm 0,2$ \\
\hline Butyrique ....... & Io,o $\pm 0,3$ & $\mathrm{I} 0, \mathrm{I} \pm 0,3$ \\
\hline Isovalérianique . & $\mathrm{r}, 3 \pm 0,3$ & $\mathrm{I}, 2 \pm 0, \mathrm{I}$ \\
\hline Valérianique .... & $0,6 \pm 0,2$ & $0,5 \pm 0,2$ \\
\hline
\end{tabular}

L'accord est bon entre les deux colonnes si l'on a pris soin de déterminer avec précision les coefficients de proportionnalité à appliquer aux réponses du détecteur aux différents acides.

En vue de diminuer 1'adsorption des acides gras volatils, de nombreux auteurs, à la suite de METCALFE (I960), ajoutèrent un acide à la phase stationnaire. Mais ce phénomène ayant lieu principalement au niveau de l'injecteur, il demeure important. Nous avons constaté que l'addition d'acide formique, à la fois au gaz vecteur (AckMAN et BURGHER I963 ; LESTER, I964) et à l'échantillon (DECKER, I962 ; CARLsTrRom et al., I965), était beaucoup plus efficace et permettait de supprimer totalement 1'adsorption lorsque les injecteurs étaient propres. FABRY (I967) met en garde contre l'introduction d'acide formique dans la phase gazeuse ; or nous opérons ainsi depuis trois ans, et $\mathbf{n}$ 'avons pas constaté le moindre dommage sur quelque partie que ce soit de l'appareil.

Pour déterminer la concentration en acides gras volatils totaux, nous disposons de deux types de méthodes : injecter des quantités bien déterminées d'échantillon et comparer les réponses à celle que donne une solution témoin (GEHRKE et LAMKIN, I96I ; ERWIN, Marco et EMery, I96I ; DF Vuyst et al., I964 ; CotTyn et BoucQue, I968) ou ajouter à l'échantillon un étalon interne (ERWIN, MARco et FMERY, I96I ; CARLstrom et al., I965; CotTyn, I966; FABRy, I967) pour lequel nous avons choisi le butanol, dont le pic n'interfère pas avec ceux des acides. Sur trois solutions connues, nous avons évalué l'acidité totale simultanément par les deux procédés afin de comparer leur précision. Nous indiquons ci-dessous, pour chaque solution analysée Io fois, la concentration exacte et la moyenne des concentrations trouvées par chacune des deux méthodes :

\begin{tabular}{|c|c|c|c|c|}
\hline & \multirow{2}{*}{$\begin{array}{c}\text { Concentration } \\
\text { exacte } \\
\text { (mmoles } / 1)\end{array}$} & \multicolumn{3}{|c|}{ Concentration trouvée (mmoles/1) } \\
\hline & & Injections & quantitatives & Etalon interne \\
\hline Solution $1 \ldots \ldots \ldots \ldots$ & 60,70 & 60,91 & $\pm 1,83$ & $60,59 \pm 1,16$ \\
\hline Solution $2 \ldots \ldots \ldots \ldots$ & 81,32 & 81,57 & $\pm 3,13$ & $81,39 \pm 1,12$ \\
\hline Solution $3 \ldots \ldots \ldots \ldots$ & 98,56 & 98,36 & $\pm 2,75$ & $98,43 \pm 1,28$ \\
\hline
\end{tabular}

La méthode basée sur l'injection quantitative de la solution inconnue et d'une solution de référence nous a donné de moins bons résultats que la méthode de l'étalon interne. Les deux raisons en sont l'impossibilité d'injecter des quantités rigoureuse- 
ment constantes et l'absence de compensation automatique des variations de sensibilité du détecteur si l'étalon n'est pas dans le mélange à analyser. A la suite de ces essais, nous avons décidé d'ajouter du butanol à nos échantillons pour déterminer les concentrations en acides gras volatils totaux. Celles-ci sont inférieures d'environ 6 p. Ioo aux valeurs obtenues par distillation. Mais il est probable que cette dernière méthode ne dose pas que les acides séparés par chromatographie.

\section{APPAREILLAGE ET MODE OPÉRATOIRE}

\section{Appareillage}

Le chromatographe est un Aerograph 200, à double colonne, muni de deux détecteurs à ionisation de flamme. Les enregistreurs " Honeywell ", de I $\mathrm{mV}$, sont équipés d'intégrateurs mécaniques "Disc".

Les colonnes d'un diamètre intérieur de $2,2 \mathrm{~mm}$, sont en acier inoxydáble et se composent de deux tronçons reliés par un raccord. Le premier tronçon mesure $2 \mathrm{~m}$ et contient du Chromosorb W, 60-80 mesh, imprégné de Carbowax $20 \mathrm{M}$ à raison de 20 p. roo de Carbowax dans le mélange. Le second, long de $50 \mathrm{~cm}$, est rempli de Porapak T, 80-10o mesh. La température du four des colonnes est de $\mathrm{I} 80^{\circ} \mathrm{C}$. Les débits des gaz sont de $30 \mathrm{ml} / \mathrm{mn}$ pour l'azote, $20 \mathrm{ml} / \mathrm{mn}$ pour l'hydrogène et $400 \mathrm{ml} / \mathrm{mn}$ pour l'air. L'azote barbote dans de l'acide formique avant de pénétrer dans l'appareil.

\section{Mode opératoire}

A $5 \mathrm{ml}$ de liquide obtenu après filtration sur mousseline d'un échantillon de contenu du rumen, on ajoute environ $0,5 \mathrm{ml}$ d'acide formique et très exactement $0,5 \mathrm{ml}$ d'une solution de butanol à $1,5 \mathrm{p}$. roo. On injectc I $\mu l$ du mélange dans l'appareil. Tous les acides jusqu'à l'acide valérianique sont élués au bout de $15 \mathrm{mn}$.

\section{Recommandations importantes}

Avec certains lots de Carbowax $20 \mathrm{M}$, nous obtenons un pic qui apparaît peu après l'injection d'un échantillon et dont la hauteur croît au cours du vieillissement de la colonne, si bien que sa traînée peut perturber le tracé du chromatogramme. Pour remédier à cet inconvénient, il suffit d'injecter sur la colonne une quantité importante d'eau $(200 \mu l)$ qui entraîne les produits de décomposition du Carbowax. Cette opération peut être effectuée sans inconvénient aussi souvent qu'il est nécessaire, par exemple, chaque matin, lors de la mise en marche de l'appareil. La colonne fonctionne, en principe, pendant plusieurs mois. Il convient d'utiliser des lots de Carbowax assez récents, car il nous est arrivé, avec certains produits anciens, de ne pouvoir chauffer la colonne au-delà de $I 5^{\circ} \mathrm{C}$ sans que la phase liquide ne soit entraînée. Ceci pourrait provenir d'une dépolymérisation partielle du Carbowax.

Après un certain temps d'utilisation de 1'appareil, une injection d'eau après le passage d'un échantillon donne lieu à la sortie d'une petite quantité de tous les acides. Ceci est dû à l'adsorption des acides gras volatils sur les impuretés qui encrassent la chambre d'injection. Il convient alors de démonter et de nettoyer les injecteurs et l'adsorption se trouve alors totalement supprimée. Dans notre laboratoire, nous procédons à cette opération une fois par semaine environ. 
L'utilisation du butanol comme étalon interne présente trois inconvénients :

- il se vaporise rapidement de la solution, ce qui oblige à le rajouter juste avant d'effectuer l'injection ;

- la sensibilité relative du détecteur au butanol par rapport à sa sensibilité aux acides gras volatils n'est pas constante, bien que sa réponse soit proportionnelle à la concentration de ces différents composés. C'est pourquoi nous effectuons des étalonnages fréquents avec une solution connue d'acide acétique dans l'eau, dans laquelle nous rajoutons du butanol. Nous injectons le mélange 4 fois le matin, 4 fois au cours de la journée et 4 fois le soir. Il y a rarement des variations au cours d'une journée, mais il est quand même bon de procéder à ces vérifications ;

- enfin, il arrive que le liquide du rumen contienne des quantités non négligeables de butanol, ce qui conduit à sous-estimer la concentration des acides gras volatils. Il convient donc, avant d'analyser les prélèvements relatifs à une expérience donnée, de passer sur la colonne un certain nombre d'échantillons afin de vérifier si le butanol est présent ou non. Dans le cas où il y en a des quantités notables, il faut déterminer 1'acidité•totale par une autre méthode (injections quantitatives).

En conclusion, cette méthode permet une bonne séparation des acides gras volatils, mais l'acidité totale est déterminée avec une précision nettement inférieure à celle que peut donner la distillation. Néanmoins, elle permet d'analyser 25 échantillons par jour sur chaque colonne et c'est un avantage considérable que de pouvoir multiplier les prélèvements, étant donné l'hétérogénéité du contenu du rumen et les variations individuelles.

Reçu pour publication en septembre 1969.

\section{SUMMARY}

\section{A TECHNIQUE FOR THE QUANTITATIVE DETERMINATION OF VOLATILE FATTY ACIDS IN RUMEN FLUID}

Satisfactory separation of ruminal volatile fatty acids was obtained by means of gas-chromatography on a Carbowax $20 \mathrm{M}$ and a Porapak T columms butt-jointed.

The reproducibility of the analysis was good.

The use of butanol as inherent labeler allowed to estimate at the same time the rates of total volatile fatty acids.

\section{RÉFÉRENCES BIBLIOGRAPHIQUES}

Ackman R.G., BURGher R. D., 1963. Quantitative gas liquid chromatographic estimation of volatile fatty acids in aqueous media. Anal. chem., 35, 647-652.

Carlstrom G., Hallgren W., Perherson B., Wallin O., I965. Gas chromatographic determination of volatile fatty acids in aqueous media. Its use in bovine rumen fluid and in silage. Acta vet. Scand., 6, 52-58.

Cotryn B. G., 1966. Dosage par chromatographie en phase gazeuse des acides gras volatils dans des extraits aqueux d'ensilages et dans le contenu de rumen. Rev. Agr. Brussels, 9, 973-983.

Cotryn B. G., Boucgue C. V., I968. Rapid method for the gas-chromatographic determination of volatile fatty acids in rumen fluid. J. Agr. Food Chem., 16, 105-107.

De Vuyst A., Vervack M., Vanbelle M., Moreels A., Arnould R., I964. Comparaison du dosage des acides gras volatils dans les ensilages et le liquide du rumen, par la méthode de chromatographie en phase gazeuse et la méthode de LePPER-FiIEG. A gricultura, 12, 233-236. 
Elsden S. R, 1946. The application of the silica gel partition chromatogram to the estimation of volatile fatty acids, Biochem. J., 40, 252-256.

EMERy E. M., Koerner W.E., I96I. Gas chromatographic determination of trace amounts of the lower fatty acids in water. Anal. Chem., 33, 146-147.

Erwin S. E., Marco G. L., EMERY E. M., I96r. Volatile fatty acids analyse of blood and rumen juice by gas chromatography. J. Dairy Sci., 44, I $768-1770$.

FABRY J., 1967. Mise au point d'une méthode d'analyse par chromatographie en phase gazeuse pour la détermination des acides gras inférieurs dans le jus de rumen. Bull. Rech. A gron. Gembloux, 2, 637-656.

Friedemann T. E., I938. The identification and quantitative determination of volatile alcohols and acids. J. Biol. Chem., 123, 161-183.

Gerrke C. W., Lamkin W. M., ig6r. Quantitative determination of steam-volatile fatty acids by gas liquid chromatography. J. Agric. Food Chem., 9, 85-88.

HUNTER I. R., ORTEGREN J. W., 1960. Gas chromatographic separation of volatile organic acids in presence of water. Anal. Chem., 32,682-684.

James A. T., Martin A. J. P., 1952. Gas-liquid partition chromatography : the separation and microestimation of volatile fatty acids from formic acid to dodecanoic acid. Biochem. J., 50, 679-690.

LeSTER D., I964. Determination of acetic acid in blood and other tissues by vacuum distillation and gas liquid chromatography. Anal. Chem., 36, I8 Io-18 I2.

Mahadevan V., Stenroos L., I967. Quantitative analysis of volatile fatty acids in aqueous solution by gas chromatography. Anal. Chem., 39, I652-1654.

Metcalfe L. D., Ig6o. Gas chromatography of unesterified fatty acids using polyester columns treated with phosphoric acid. Nature, 188, 142-143.

MONK P. R., FORREst W. W., r967. Sample introduction in analysis of volatile fatty acids in rumen fluid by gas chromatography. J. Chromatogr. Netherl., 30, 203.

Storry J. E., Millard D., I 965 . The determination of steam-volatile fatty acids in rumen liquor, blood plasma and milkfat. J. Sci. Food A gric., 16, 417-420.

Tilley J. M. A., Canaway R. J., Terry R. A., I964. The estimation of volatile fatty acids by gas chromatography with automatic titration. Analyst, 89. 363-365.

VANDENheUVEL F. A., I964. Rapid, accurate microanalysis of the lower fatty acids with particular reference to serial determination Anal. Chem., 36, I930-I934.

Van Eenaeme C. G., Bienfait J. M., Lambot O., 1965 . La détermination quantitative des acides gras volatils dans le liquide du rumen par chromatographie en phase gazeuse. Ann. Méd. Vét., 8, 569584 . 